\title{
Experimental Study on the Impact of Regenerator Placement Strategies when Dynamically Provisioning in Translucent GMPLS WSON Networks
}

\author{
R. Martínez ${ }^{1}$, R. Casellas ${ }^{1}$, R. Muñoz ${ }^{1}$, B. García-Manrubia ${ }^{2}$, P. Pavón-Marino ${ }^{2}$, M. Klinkowski ${ }^{3}$, D. Careglio ${ }^{4}$ \\ ${ }^{1}$ Centre Tecnologic de Telecomunicacions de Catalunya (CTTC), Av. Carl Friedrich Gauss n7, 08860 Castelldefels, Spain \\ ${ }^{2}$ Universidad Politécnica de Cartagena (UPCT), Plaza del Hospital 1, 30202, Cartagena, Spain \\ ${ }^{3}$ National Institute of Telecommunications, 1 Szachowa Street, 04-894 Warsaw, Poland \\ ${ }^{4}$ Universtiat Politècnica de Catalunya (UPC), Jordi Girona 1-3, 08034, Barcelona, Spain \\ \{ricardo.martinez, ramon.casellas,raul.munoz\}@cttc.es; \{belen.garcia,pablo.pavon\}@upct.es; mklinkow@tl.waw.pl; careglio@ac.upc.edu
}

\begin{abstract}
The impact of the regenerator placement strategies when dynamically provisioning connections in translucent WSON is studied. The evaluation is experimentally conducted in the ADRENALINE testbed in terms of the connection blocking.

OCIS codes: (060.4510) Optical communications; (060.4251) Networks, assignment and routing algorithms.
\end{abstract}

\section{Introduction}

Optical network architectures are evolving from traditional opaque networks, in which optical signal undergoes an optical-electrical-optical $(\mathrm{O} / \mathrm{E} / \mathrm{O})$ regeneration, toward all-optical (i.e., transparent) networks. The lack of practical all-optical regeneration gives rise to the so called translucent (or semi-transparent) network architectures, in which a set of sparsely but strategically placed $\mathrm{O} / \mathrm{E} / \mathrm{O}$ regenerators is used to maintain the acceptable level of the signal quality [1]. Moreover, an electrical regenerator enables the possibility of wavelength conversion which may help to decrease the number of rejected connection requests due to the wavelength continuity constraint (WCC). In this context, translucent Wavelength Switched Optical Networks (WSONs) have been the first network infrastructure to receive the attention from the research community, principally, due to the maturity of the technology. Indeed, protocol extensions and requirements to take into account the presence of physical-layer impairments (PLIs) in WSONs are currently under development within IETF [2]. In addition, in [3], translucent-oriented control plane protocol extensions within the Generalized Multi-Protocol Label Switching (GMPLS) framework, similar to those being discussed within IETF WSON [2], have been experimentally validated.

In translucent WSONs, there is the problem of the regenerator placement (RP) and the regenerator allocation (RA), which refer to planning and operational phase respectively. A common approach is to apply firstly a RP algorithm to sparsely and strategically place the regenerators throughout the network using a static set of connections (i.e., a given static/forecasted traffic demands), and afterwards a RA algorithm assigns the already placed regenerators to the incoming dynamic connection requests under the constraint of acceptable optical signal quality. RP is an offline problem and aims at reducing the number of regenerators, that is the network cost; in contrast, RA is an online problem whose goal is to minimize the utilization of the regenerators, and thus, minimize the connection blocking. In this paper we focus on experimentally study the impact of the RP phase when subject to dynamic provisioning in a translucent GMPLS WSON. Particularly, we apply a common RA algorithm and compare different RP algorithms.

\section{Considered Translucent Regenerator Placement Strategies and On-line Routing Algorithm}

The RP strategies proposed in the literature take advantage of available information about the physical topology and/or the traffic prediction of the network to perform the RP. In our case, we study and implement two algorithms based on network topology: Nodal Degree First (NDF) and Centered Node First (CNF) [4]; and one algorithm which uses traffic prediction: sLERP [5]. In NDF regenerators are placed at nodes which have a higher nodal degree. In $\mathrm{CNF}$, the selected nodes to place the regenerators are the most centered, i.e., the nodes traversed by a greater number of shortest hop-distance paths in the network. Alternatively, sLERP algorithm is composed of two phases. The first one computes RWA (Routing Wavelength Assignment) using a traffic prediction. The second phase obtains the degradation level of each previously routed demand and places the regenerators where is necessary according to the degradation threshold following the trace-back strategy proposed in [4].

Additionally to the above algorithms, we propose an ILP-based algorithm: PS-BR (Path Segmentation with Blocking and Regenerator usage objective). It makes use of pre-defined end-to-end paths that are decomposed onto transparent lightpath segments. A regenerator is placed between two segments. The optimization objective minimizes, firstly, the number of blocked lightpaths and, secondly, the number of regenerators used. PS-BRW extends PS-BR which apart from the previous objectives minimizes the network overall wavelength usage. 
Once the RP problem is solved, a RWA algorithm together with the RA dynamically computes the route for each incoming connection (Label Switched Path - LSP - in the GMPLS context) and assigns regenerators when required. To this end, we rely on the online Optical Signal Noise Ratio (OSNR)-based path computation for translucent WSON presented in [3]. This RWA/RA algorithm uses a link- and a node-cost model function that computes the shortest path satisfying two restrictions: R1, dealing with the WCC; and R2, computing routes with adequate optical signal quality at the receiver. Additionally, the RWA/RA algorithm aims at minimizing the amount of used network resources (i.e., wavelengths and regenerators) to favor the establishment of future LSP requests. In this work, the link-cost is set to 1 as long as unused wavelengths are available on a link. This allows balancing the traffic load through all the network links, and thus achieves an efficient usage of all the wavelengths. By doing so, the WCC (R1) is well addressed avoiding the exhaustion of particular links. On the other hand, the node-cost is also set to 1. Since the regenerators are, in general, scarcer than the wavelengths on a link, it is preferable to occupy more wavelength links than allocating an available regenerator at a node. For the sake of clarification, for a given sourcedestination pair, if two feasible routes span the same number of links but different number of regenerators, the selected route is the one with the shortest path cost, i.e., the path using less regenerators.

\section{Experimental Evaluation}

The performance evaluation is carried out within the ADRENALINE testbed at CTTC's Lab, focusing on the LSP blocking as the metric. The considered network topology follows a European mesh network formed by 24 links and 14 optical nodes (Fig. 1. a). Regenerators are placed according to the set of RP algorithms considered in the test. Each optical link consists of two standard single mode fibers (SSMF) supporting 32 wavelengths per direction and has a variable number of spans of $80 \mathrm{~km}$ each. A span has an optical amplifier used to compensate the power loss on the optical signal due to the fiber and node attenuations. The increased number of amplifiers makes the Amplifier Spontaneous Emission (ASE) a significant impairment factor captured in the OSNR. In our model, we estimate the OSNR as the Quality of Transmission (QoT) parameter to evaluate the feasibility of the transparent segments [3]. Specifically, the total OSNR $\left(O S N R_{\text {total }}\right)$ is compared to a threshold level $\left(O S N R_{t h r}\right)$ to determine whether the QoT parameter is fulfilled. If not, an available $3 \mathrm{R}$ regenerator is allocated at an intermediate node to improve the signal quality. Additionally to the ASE noise, other PLIs such as PMD, CD and the nonlinearities may impact on the signal quality. These PLIs are integrated into the constraint model as OSNR penalties provided that the signal degradation caused by them is kept within acceptable ranges [3]. Two components contribute to the OSNR level estimation in a transparent segment traversing $l$ links: the link OSNR $\left(O S N R_{\text {link }}\right)$ considering the ASE noise due to the pre- and line amplifier spans of a link, and the node OSNR $\left(O S N R_{\text {node }}\right)$ considering the ASE noise caused by the booster amplifier. Both the $O S N R_{\text {link }}$ and $O S N R_{\text {node }}$ values are computed offline using the parameters defined in Fig. 1. a.

The regenerator placement algorithms studied have been implemented in MATLAB code, integrated and tested in the MatPlanWDM tool [6]. The parameters used in NDF and CNF algorithms are 6 nodes as regeneration capable nodes and 8 regenerators are placed at each node. The factors used in SLERP are: (i) the number of shortest paths computed associated to each demand is set to 4, (ii) the black list size is set to 100, and (iii) the number of permutations performed in the demand set is 1000. In PS-BR and PS-BRW the value selected for the number of shortest paths, considering the physical distance, is 4 between each pair of nodes. The results obtained using the previous information are shown in Table 1.

Table 1. Results of the regenerator placement strategies

\begin{tabular}{|l|c|c|c|c|c|c|c|c|c|c|c|c|c|c|c|}
\hline Algorithm\Node & $\mathrm{N}_{1}$ & $\mathrm{~N}_{2}$ & $\mathrm{~N}_{3}$ & $\mathrm{~N}_{4}$ & $\mathrm{~N}_{5}$ & $\mathrm{~N}_{6}$ & $\mathrm{~N}_{7}$ & $\mathrm{~N}_{8}$ & $\mathrm{~N}_{9}$ & $\mathrm{~N}_{10}$ & $\mathrm{~N}_{11}$ & $\mathrm{~N}_{12}$ & $\mathrm{~N}_{13}$ & $\mathrm{~N}_{14}$ & Total \\
\hline CNF & 8 & 8 & 0 & 0 & 8 & 0 & 8 & 8 & 8 & 0 & 0 & 0 & 0 & 0 & $\mathbf{4 8}$ \\
\hline NDF & 8 & 0 & 0 & 0 & 8 & 8 & 0 & 0 & 8 & 8 & 0 & 0 & 8 & 0 & $\mathbf{4 8}$ \\
\hline sLERP & 0 & 0 & 0 & 0 & 5 & 2 & 2 & 7 & 5 & 1 & 0 & 0 & 6 & 0 & $\mathbf{2 8}$ \\
\hline PS-BR & 0 & 0 & 0 & 0 & 2 & 2 & 3 & 6 & 8 & 5 & 0 & 0 & 2 & 0 & $\mathbf{2 8}$ \\
\hline PS-BRW & 0 & 0 & 0 & 0 & 4 & 5 & 3 & 4 & 7 & 3 & 0 & 0 & 3 & 0 & $\mathbf{2 8}$ \\
\hline
\end{tabular}

The main assumptions for the evaluation are: the LSP-arrival process is Poisson (average time of $3 \mathrm{~s}$ ) and the LSP holding time follows a negative exponential distribution. Each data point is obtained requesting 15k LSPs. Fig. 1.b plots the LSP blocking versus the network offered load (Erlangs) for the transparent network (i.e., no regenerators) and for the considered RP strategies. It is worth noting that an LSP is blocked when the RWA/RA algorithm fails to satisfy either R1 or R2 constraints Furthermore, the signaling mechanism may also block an LSP if resource contention (i.e., regenerators or wavelengths) among different LSPs being set up occurs.

We observe that the transparent scenario performs the worst for all the traffic loads due to the unacceptable optical signal quality (i.e., $O S N R_{\text {total }}<O S N R_{\text {thr }}$ ) in the large routes. Indeed, there are a specific set of node pairs for which no feasible LSP, in terms of the optical signal quality, exists. Thereby, a lower bound of the connection blocking regardless of the offered traffic load can be computed. This lower bound is approximately 0.164 when the traffic is 


\section{OThAA3.pdf}

uniform between all node pairs (30 out of the 182 possible LSPs are unfeasible). Obviously, as regenerators are placed significant blocking reductions are attained. The reason behind this is that using a regenerator allows, on the one hand, regenerating the optical signal when needed and, on the other hand, reducing the blocking caused by the WCC. For the evaluated RP strategies, the lower connection blocking is achieved by the CNF scheme using 48 regenerators. This scheme aims at placing the pool of regenerators at the network nodes being more centered. Thus, since the used RWA/RA algorithm establishes the LSPs through the shortest paths as long as R1 and R2 are met, the regenerators of the centered nodes tend to be highly used. The NDF scheme also places 48 regenerators but the selected nodes by the RP algorithm have the higher nodal degree. Observe that the LSP blocking attained by this RP scheme performs worse than the CNF (up to 60\%): some of the regenerators placed by the NDF scheme are barely utilized since the respective nodes are not traversed by most of the shortest paths. The sLERP, PS-BR and PS-BRW RP strategies require less regenerators (28) with respect to the NDF and CNF schemes. Nevertheless, they attain a blocking performance significantly (at low traffic loads) better than the NDF scheme. The blocking differences among these three RP strategies are almost negligible. In light of the above, one may state that more sophisticated RP strategies such as sLERP, PS-BR and PS-BRW yield a better trade-off between the network cost (due to the regenerators) and the service provisioning performance (the connection blocking). Finally, note that as the traffic load grows the differences among all the RP strategies tend to disappear. Specifically, at these traffic loads, the main restriction is no longer the optical signal quality (R2) but the lack of available wavelengths (R1).

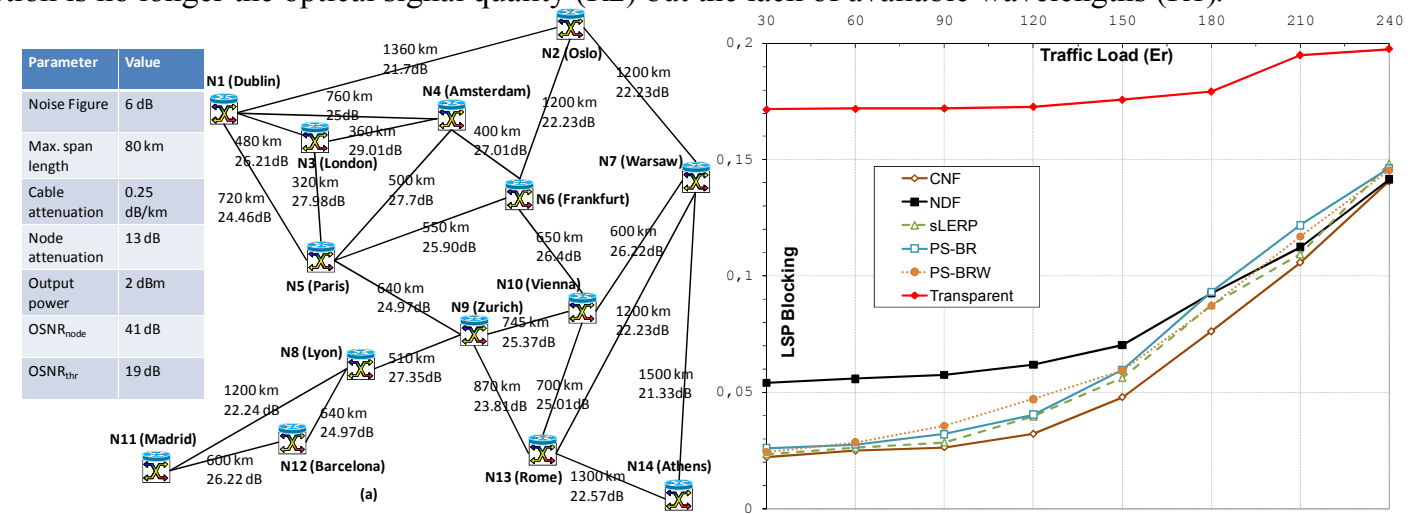

Fig. 1. a. Network topology: link distance $(\mathrm{km})$, link $\operatorname{OSNR}(\mathrm{dB})$ and physical parameters; b. Connection blocking probability.

\section{Conclusions and further work}

The RP and RA problems of translucent WSON are experimentally addressed in a GMPLS network. For a European mesh network, selected RP strategies are used resulting in different translucent networks which differ on both the number and node location of the regenerators. Then, using an online OSNR-based RWA/RA algorithm, the LSPs are dynamically provisioned on each these networks. The performance evaluation is carried out within the ADRENALINE testbed and consists in comparing the LSP blocking attained on each translucent network. From the results, we observe the significant impact of the RP strategies especially at the low traffic loads which states a tradeoff between the network cost (due to the regenerators) and the network performance (e.g., LSP blocking).

\section{Acknowledgements}

This work was partially funded by the MICINN (Spanish Ministry of Science and Innovation) through the project DORADO under contract TEC2009-07995, and developed within the framework of the BONE-project ("Building the Future Optical Network in Europe"), a Network of Excellence funded by the European Commission through the 7th ICT-Framework Programme.

\section{References}

[1] Shen and R. S. Tucker, "Translucent optical networks: the way forward," IEEE Commun. Mag., vol. 45 (2), pp. 48-54, Feb. 2007.

[2] Y. Lee, G. Bernstein, D. Li, G. Martinelli, "A Framework for the Control of Wavelength Switched Optical Networks (WSON) with Impairments," IETF draft, draft-ietf-ccamp-wson-impairments (work in progress), May 2010.

[3] R. Martínez et. al., "Experimental translucent-oriented routing for dynamic lightpath provisioning in GMPLS-enabled wavelength switched optical networks," IEEE/OSA Journal of Lightwave Technology, vol. 28 (8), pp. 1241-1255, April 2010.

[4] Xi Yang, Byrav Ramamurthy, "Sparse Regeneration in Translucent Wavelength-Routed Optical Networks: Architecture, Network Design and Wavelength Routing," Photonic Network Communications, vol. 10 (1), pp. 39-53, July 2005.

[5] M. A. Ezzahdi, et. al, "LERP: A Quality of transmission dependent heuristic for routing and wavelength assignment in hybrid WDM networks," in Proc. of ICCCN 2006, Virginia, USA, 2006, pp. 125-136.

[6] P. Pavon-Marino, et. al, "MatPlanWDM: An educational tool for network planning in wavelength-routing networks," in Proc. of ONDM 2007, Athens, Greece, 2007, pp. 58-67. 